\title{
Fullerene fine particles adhere to pollen grains and affect their autofluorescence and germination
}

This article was published in the following Dove Press journal:

Nanotechnology, Science and Applications

19 May 2011

Number of times this article has been viewed

\author{
Hideki Aoyagi \\ Charles U Ugwu \\ Life Science and Bioengineering, \\ Graduate School of Life and \\ Environmental Sciences, University \\ of Tsukuba, Tsukuba, Japan
}

\begin{abstract}
Adhesion of commercially produced fullerene fine particles to Cryptomeria japonica, Chamaecyparis obtusa and Camellia japonica pollen grains was investigated. The autofluorescence of pollen grains was affected by the adhesion of fullerene fine particles to the pollen grains. The degree of adhesion of fullerene fine particles to the pollen grains varied depending on the type of fullerene. Furthermore, germination of Camellia japonica pollen grains was inhibited by the adhesion of fullerene fine particles.
\end{abstract}

Keywords: Cryptomeria japonica, Chamaecyparis obtusa, Camellia japonica, autofluorescence, pollen grains, fullerene fine particle

\section{Introduction}

Nanotechnology is now being utilized all over the world, both in research, and in practical applications. Nanosized fine particles are of increasing importance because they are suitable for many applications in the fields of electronics, ${ }^{1}$ sensors,${ }^{2}$ catalysis, ${ }^{3}$ medicine, ${ }^{4}$ and biotechnology. ${ }^{5-7}$ Production of nanosized fine particles such as fullerene, titanium dioxide, and carbon nanotubes, has increased rapidly in recent years. Large quantities of these fine particles are discharged to the environment intentionally or unintentionally in the course of their production, use, and disposal. These will inevitably lead to pollution of both the biotic and abiotic components of the environment. Studies on the potential effects of these fine particles on human health and on the environment are therefore very important. ${ }^{8-14}$

It was reported that various fine particles adhered to pollen in the atmosphere. ${ }^{15,16}$ The physiological activities of pollen grains could be influenced by the adhesion of fine particles. Speranza et $\mathrm{al}^{14}$ reported that Pd-nanoparticles cause increased toxicity to kiwifruit pollen compared to soluble Pd (II). However, there is as yet no report on the adhesion of pollen grains and fullerene fine particles. Fullerene nanoparticles frequently form fine aggregates, so we describe them here as "fullerene fine particles". In this study, adhesive characteristics between pollen grains and fullerene fine particles and the effects of fullerenes on the germination of pollen grains were investigated.

\section{Materials and methods}

Pollen grains

Pollen grains of Cryptomeria japonica, Chamaecyparis obtusa and Camellia japonica were used as a model (these pollen grains cause hay fever in Japan). Pollen grains of Cryptomeria japonica and Chamaecyparis obtusa were purchased from the 
Genetic Resource Department, Forest Tree Breeding Center (Ibaraki, Japan). Pollen grains of Camellia japonica were obtained from the botanical gardens of the University of Tsukuba. These were dehydrated using silica gel and stored at $-80^{\circ} \mathrm{C}$.

\section{Chemicals}

Fullerene fine particles were used as a model for commercially produced fine particles. Many kinds of fullerene fine particles are commercially produced and widely used for various applications. For our experiments, two types of fullerene fine particles were purchased: $\mathrm{F} 1\left(\mathrm{C}_{60} / \mathrm{C}_{70}\right.$ mixture containing $20 \% \mathrm{C}_{70}$ and $1 \%$ higher fullerenes) from Strem Chemicals (Newburyport, MA) and F2 ( $\left.\mathrm{C}_{60} 99 \%\right)$ from Honjo Chemical Co (Osaka, Japan). The stored pollen grains of Cryptomeria japonica, Chamaecyparis obtusa and Camellia japonica were maintained at $4^{\circ} \mathrm{C}$ for 4 hours and then at $25^{\circ} \mathrm{C}$ for 1 hour before mixing with fullerene. Five $\mathrm{mg}$ of each kind of pollen grains were placed in $2 \mathrm{~mL}$ Eppendorf tubes, followed by addition of $2 \mathrm{mg}$ each of F1 and F2, then mixed using a vortex mixer for 150 seconds. After mixing, they were placed on glass slides (Matunami Glass Co Ltd, Osaka, Japan).

\section{Microscopic observations}

We have previously developed a method for identifying different pollen grains on the basis of autofluorescence and size of pollen grains. ${ }^{17,18}$ Fluorescence and bright-field micrographs of pollen grains on a glass plate were taken using a fluorescence microscope (Leica DMR; Leica Microsystems, Tokyo, Japan) equipped with both an ultraviolet illuminating system using a xenon lamp and a band-pass filter (central wavelength: $340 \mathrm{~nm}$ ) and CCD camera (SPOT ISA-CE Color; Diagnostic Instruments, Inc, Sterling Heights, MI). Samples for scanning electron microscopy study were prepared according to the method of Vinckier and Smets. ${ }^{19}$ Samples were observed with a field emission scanning electron microscope (JSM-6330F; JEOL, Tokyo, Japan).

\section{Germination efficiency}

The effect of the adhesion of fullerene fine particles on the germination of pollen grains was studied using Camellia japonica pollen grains (the germination conditions for Camellia japonica pollen grains have already been determined ${ }^{20}$ but the conditions for Cryptomeria japonica and Chamaecyparis obtusa have not been determined yet). Stored pollen grains of Camellia japonica were maintained at $4^{\circ} \mathrm{C}$ for 4 hours and then at $25^{\circ} \mathrm{C}$ for 1 hour before mixing with fullerene and commencing germination tests. Five mg of pollen grains of Camellia japonica were placed in a $2 \mathrm{~mL}$ Eppendorf tube, followed by addition of $2 \mathrm{mg}$ of each type of fullerene fine particle and mixed using a vortex mixer for 150 seconds. After mixing, the mixture was placed on a sterilized agar medium ${ }^{20}$ (which consisted of 1.3\% agar, 10\% sucrose, autoclaved at $121^{\circ} \mathrm{C}$ for 15 minutes) on top of a glass slide. Cultures of pollen grains were carried out at $25^{\circ} \mathrm{C}$. After 24 hours of incubation, the percentage of germinated pollen grains was calculated. The experiments were performed in triplicate.

\section{Results and discussion}

Figure 1 shows fluorescence micrographs for adhesion of both F1 and F2 fullerenes to Cryptomeria japonica pollen grains. Autofluorescence decreased when F1 fullerenes adhered to pollen grains (Figure 1c) and it was more affected by F1 than F2 fullerenes (Figure 1d). Figure 2 shows fluorescence micrographs of adhesion between pollen grains of Chamaecyparis obtusa and fullerene fine particles (F1 and F2). Fluorescence micrographs for the adhesion of pollen grains of Camellia japonica with F1 and F2 are shown in Figure 3.

As with Cryptomeria japonica, autofluorescence of Chamaecyparis obtusa and Camellia japonica was reduced by adhesion of F1 (Figures 2c and 3c). The pollen grains of Chamaecyparis obtusa and Camellia japonica showed more reduction in autofluorescence with F1 than with F2 adhesion. When pollen grains of Cryptomeria japonica, Chamaecyparis obtusa or Camellia japonica with adhered F1 or F2 were added to $0.6 \mathrm{M}$ mannitol solution (isotonic solution) and gently suspended, some of the F1 or F2 detached from the
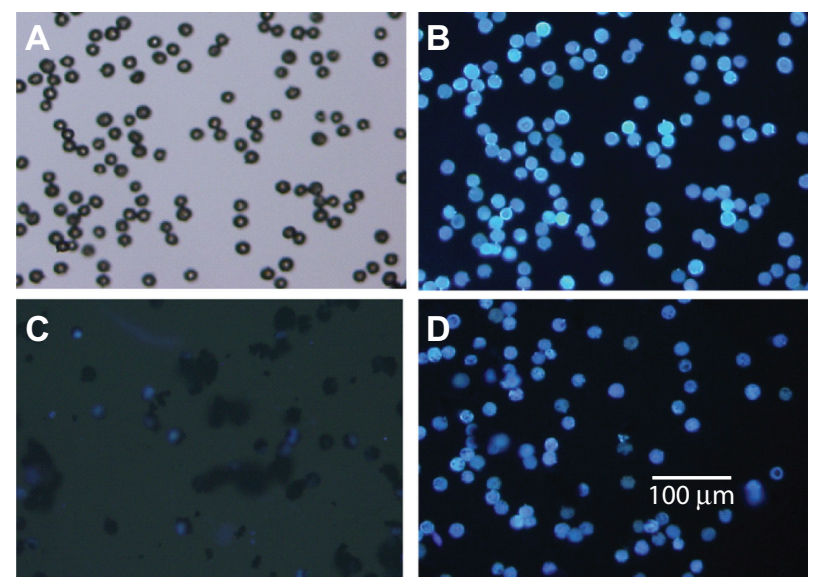

Figure I Adhesion of various fullerene fine particles to Cryptomeria japonica pollen grains. A) No addition [light microscope], B) No addition [fluorescence microscope], C) Addition of fullerene ( $\mathrm{FI}$ ) fine particles [fluorescence microscope], D) Addition of fullerene (F2) fine particles [fluorescence microscope]. 

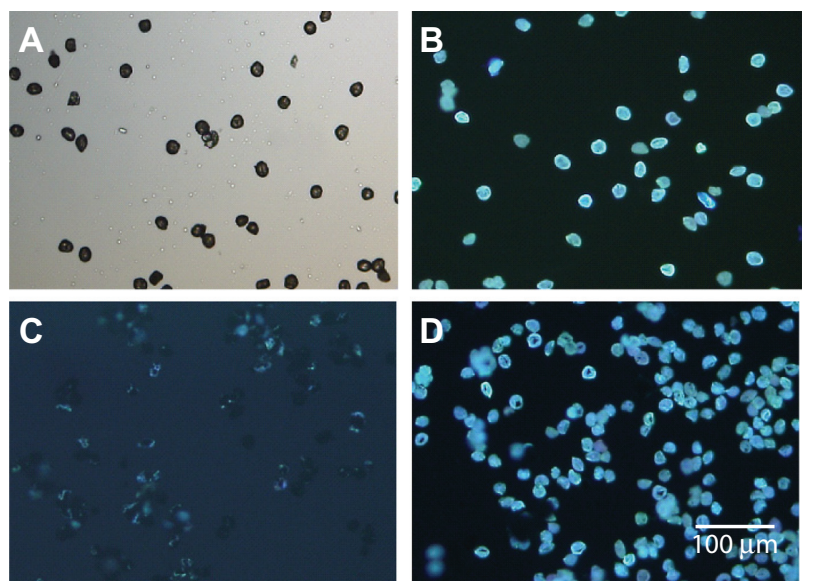

Figure 2 Adhesion of various fullerene fine particles to Chamaecyparis obtusa pollen grains. A) No addition [light microscope], B) No addition [fluorescence microscope], C) Addition of fullerene (FI) fine particles [fluorescence microscope], D) Addition of fullerene (F2) fine particles [fluorescence microscope].

pollen grains, and their autofluorescence was observed again under a fluorescence microscope (data not shown). It was confirmed that F1 and F2 did not destroy the autofluorescing substance but reduced its activity by adhering to the pollen grains. Characteristics of fullerene fine particles such as their size distribution, surface structure, specificity, and components may affect adhesion (the mechanisms and detailed characteristics of $\mathrm{F} 1$ and $\mathrm{F} 2$ are now being investigated).

Adhesion between pollen grains and fullerene fine particles could be attributed to their surfaces (ie, structure and composition) and the extent of their compatibility with one another. The surface of pollen grains of Cryptomeria japonica, Chamaecyparis obtusa or Camellia japonica differed from one another (Figure 4). Also, the pollen grains have wide variation in the composition and structure of
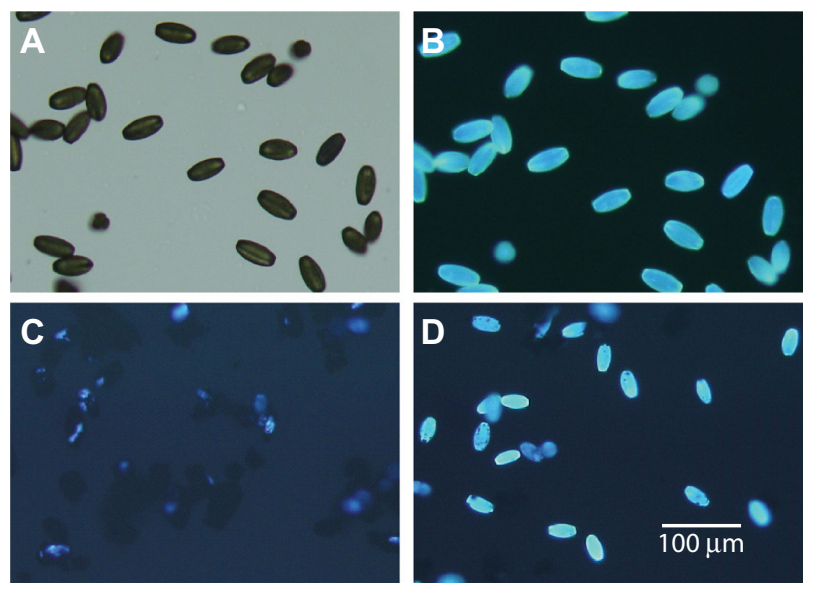

Figure 3 Adhesion of various fullerene fine particles to Camellia japonica pollen grains A) No addition [light microscope], B) No addition [fluorescence microscope], C) Addition of fullerene ( $\mathrm{FI}$ ) fine particles [fluorescence microscope], D) Addition of fullerene (F2) fine particles [fluorescence microscope].
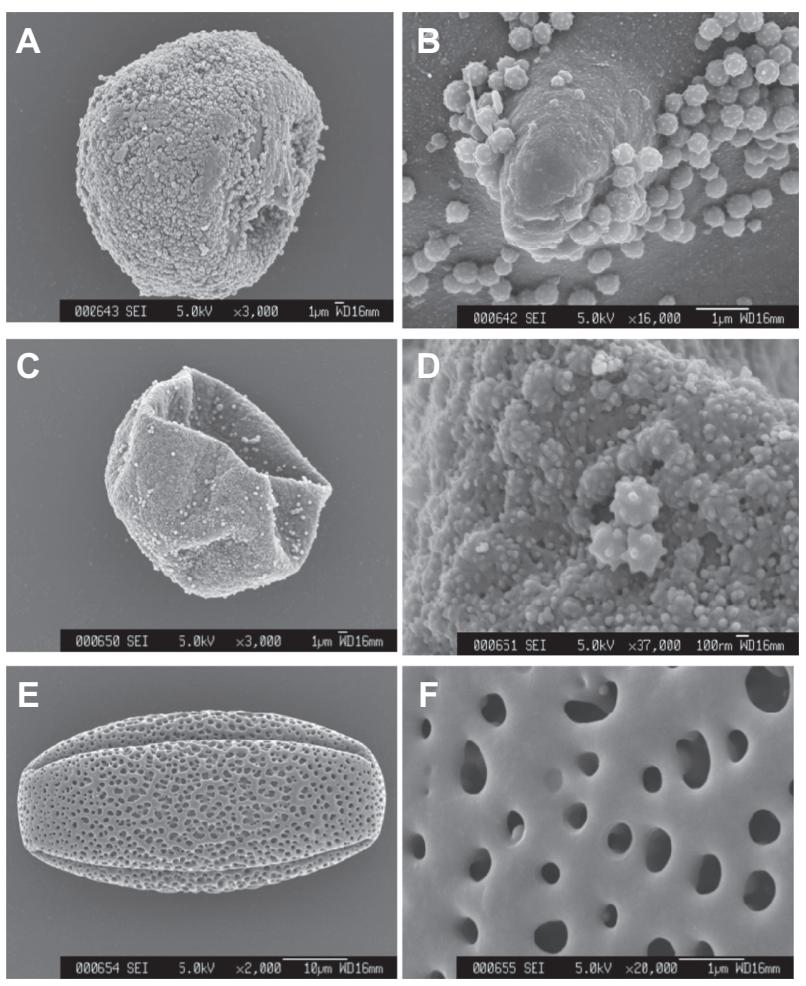

Figure 4 Scanning electron microscopic images of various pollen grains. A) Cryptomeria japonica pollen grain, B) Surface of Cryptomeria japonica pollen grain, C) Chamaecyparis obutsa pollen grain, D) Surface of Chamaecyparis obutsa pollen grain, E) Camellia japonica pollen grain, F) Surface of Camellia japonica pollen grain.

their outer walls (orbicle and sexine for the outermost layer; nexine for the innermost layer) and inner walls. ${ }^{19}$ The cell walls of pollen grains are mainly composed of sporopollenin, lipids, lignin and other components. ${ }^{21-23}$ On the other hand, some reports have shown that aside from the composition of carbon black and polystyrene nanosized fine particles, extremely small particle size, surface area, surface structure, and oxidizing capacity affect their properties. ${ }^{24,25}$

The effect of the adhesion of F1 and F2 fullerene fine particles on the germination of Camellia japonica pollen grains is shown in Figure 5(a-c). The influence of F1 and F2 fullerene fine particles on the germination ratio of Camellia japonica pollen grains is shown in Figure 5(d). The germination ratio of Camellia japonica pollen grains with adhesion of F1 was decreased by one-third compared with the control. The viability of the Camellia japonica pollen grains was estimated at about $90 \%$ using a fluorochromatic reaction test ${ }^{26,27}$ (data not shown). Possibly the adhesion of F1 to pollen grains causes damage to the plasma membrane. In order to understand why F1 obstructed the germination of pollen grains of Camellia japonica, comparative evaluation of F1 and F2 properties such as surface area, surface structure, oxidation potentials, and their radical scavenging 

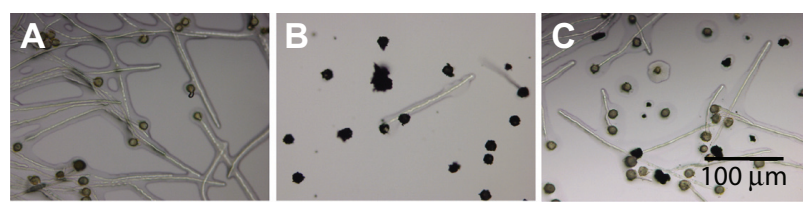

D

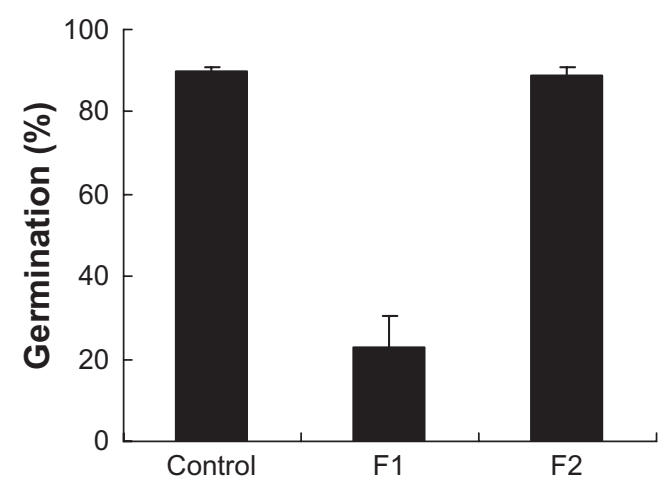

Figure 5 Effect of the adhesion of various fullerene fine particles to $C$. japonica pollen grains on their germination. A) No addition, B) Addition of fullerene (FI) nanoparticles, C) Addition of fullerene (F2) fine particles, D) Germination ratio. The error bars show the standard deviation of the mean value of three samples.

activities have been considered, and studies of these aspects of the fullerenes are now underway.

Here we report for the first time, an analysis of the adhesion characteristics of fullerene fine particles to pollen grains, and the effects of these particles on the germination of pollen grains. When evaluating adverse effects in the practical application of fullerene fine particles, selection of those that have little effect on living organisms and the environment would be desirable. However, it should be noted that the work presented here is qualitative analysis of adhesion between fullerene fine particles and pollen grains. Quantitative evaluation of the degree of adhesion (or adhesion efficiency) between fullerene fine particles and pollen grains is yet to be conducted.

Roshchina and Karnaukhov ${ }^{28}$ investigated changes in the autofluorescence spectra of Philadelphus grandiflorus, Epiphyllum hybridum, and Plantago major pollen grains after 100 hours exposure to ozone. The fluorescence maximum at 530-550 nm disappeared in carotenoid-containing pollen grains of Philadelphus grandiflorus and Epiphyllum hybridum, and a new maximum at $475-480 \mathrm{~nm}$ arose that correlated with lipofuscin-like substances observed in the extracts from the pollen grains. The carotenoid-less pollen of Plantago major showed only an increase of the maximum at $470 \mathrm{~nm}$, and no lipofuscin in the extracts (the main indicators of pollen damage by ozone in carotenoid-containing microspores are the lipofuscin-like compounds).

It is necessary to analyze various pollen grains and many kinds of fullerene fine particles to be able to quantify their degree of adhesion. Where fullerene fine particles are responsible for oxidation and radical activities, ${ }^{29,30}$ the degree of tolerance of the fullerene particles by the pollen grains may be related to the antioxidative potential of the pollen grains. Pollen grains exhibit autofluorescence by amino acids, vitamins, isoprenoids (carotenoids), phenyl propanoids (lignin and flavonoids), and quinones. The relationship between the fullerene fine particles and the antioxidative potential of various pollen grains, as well as their effect on the germination of pollen grains, will be examined. We are also currently investigating the adhesion characteristics between various kinds of other fine particles (titanium dioxide, carbon nanotubes) and various kinds of pollen grains, and their effects on autofluorescence and germination.

\section{Acknowledgment}

This work was supported by a Grant-in-aid for Plant Research (The New Technology Development Foundation, Kitamagome, Otaku, Tokyo, Japan).

\section{Disclosure}

The authors report no conflicts of interest in this work.

\section{References}

1. Nagasawa H, Ichimura A, Isoda S. Carboxylate-passivated silver nanoparticles and their application to sintered interconnection: a replacement for high temperature lead-rich solders. J Electron Mater. 2010;39: 1233-1240.

2. Liu SQ, Tang ZY. Nanoparticle assemblies for biological and chemical sensing. J Mater Chem. 2010;20:24-35.

3. Narayanan R. Recent advances in noble metal nanocatalysts for Suzuki and Heck cross-coupling reactions. Molecules. 2010;15:2124-2138.

4. Cortivo R, Vindigni V, Lacobellis L, Abatangelo G, Pinton P, Zavan B. Nanoscale particle therapies for wounds and ulcers. Nanomedicine. 2010;5:641-656.

5. West JL, Halas NJ. Applications of nanotechnology to biotechnology commentary. Curr Opin Biotechnol. 2000;11:215-217.

6. Penn SG, He L, Natan MJ. Nanoparticles for bioanalysis. Curr Opin Chem Biol. 2003;7:609-615.

7. Liu WT. Nanoparticles and their biological and environmental applications. J Biosci Bioeng. 2006;102:1-7.

8. Dreher KL. Heath and environmental impact on nanotechnology: toxicological assessment of manufactured nanoparticles. Toxicol Sci. 2004;77:3-5.

9. Oberdorster G, Oberdorster E, Oberdorster J. Nanotoxicology: An emerging discipline evolving from studies of ultrafine particles. Environ. Health Perspect. 2005;113:823-839.

10. Porter AE, Gass M, Muller K, Skepper JN, Midgley P, Welland M. Visualizing the uptake of $\mathrm{C}_{60}$ to the cytoplasm and nucleus of human monocyte-derived macrophage cells using energy-filtered transmission electron microscopy and electron tomography. Environ Sci Technol. 2007;41:3012-3017.

11. Baun A, Sorensen SN, Rasmussen RF, Hartmenn NB, Koch CB. Toxicity and bioaccumulation of xenobiotic organic compounds in the presence of aqueous suspensions of aggregates of nano- $\mathrm{C}_{60}$. Aquat Toxicol. 2008;86:379-387.

12. Wang J, Zhang X, Chen Y, Sommerfeld M, Hu Q. Toxicity assessment of manufactured nanomaterials using the unicellular green alga Chlamydomonas reinhardtii. Chemosphere. 2008;73:1121-1128. 
13. Velzeboer I, Hendrix AJ, Ragas AM, van de Meent D. Aquatic ecotoxicity tests of some materials. Environ Toxicol Chem. 2009;27: 1942-1947.

14. Speranza A, Leopold K, Maier M, Taddei AR, Scoccianti V. Pdnanoparticles cause increased toxicity to kiwifruit pollen compared to soluble Pd (II). Environ Pollut. 2010;158:873-882.

15. Okuyama Y, Matsumoto K, Okochi H, Igawa M. Adsorption of air pollutants on the grain surface of Japanese cedar pollen. Atmospheric Environ. 2007;41:253-260.

16. Guedes A, Ribeiro N, Oliveria M, Noronha F, Abreu I. Comparison between urban and rural pollen of Chenopodium alba and characterization of adhered pollutant aerosol particles. Aerosol Sci. 2009;40: 81-86.

17. Mitsumoto K, Yabusaki K, Aoyagi H. Classification of pollen grainspecies using autofluorescence image analysis. J Biosci Bioeng. 2009;107: 90-94.

18. Mitsumoto K, Yabusaki K, Kobayashi K, Aoyagi H. Development of a novel real-time pollen grainsorting counter using species-specific pollen autofluorescence. Aerobiologia. 2010;26:99-111.

19. Vinckier S, Smets E. The potential role of orbicules as a vector of allergens. Allergy. 2001;56:1129-1136.

20. Hasegawa Y, Nakamura S, Nakamura N. Immunocytochemical localization of callose in the germinated pollen of Camellia japonica. Protoplasma. 1996;194:133-139.

21. Uehara K, Sahashi N. Pollen wall development in Cryptomeria japonica (Taxodiaceae). Grana. 2000;39:267-274.

22. Yatomi R, Nakamura S, Nakamura N. Immunochemical and cytochemical detection of wall components of germinated pollen of gymnosperms. Grana. 2002;41:21-28.
23. Majewska-Sawka A, Fernandez MC, M'rani-Alaoui M, Munster A Rodriguez-Garcia MI. Cell wall reformation by pollen tube protoplasts of olive (Olea europaea L.): structural comparison with the pollen tube wall. Sex Plant Reproduct. 2002;15:21-29.

24. Stone V, Shaw J, Brown DM, MacNee W, Faux SP, Donaldson K. The role of oxidative stress in the prolonged inhibitory effect of ultrafine carbon black on epiyhelial cell function. Toxicol in Vitro. 1998;12: 649-659.

25. Brown DM, Wilson MR, Macnee W, Stone V, Donaldson K. Sizedependent proinflammatory effects of ultrafine polystyrene particles: A role for surface area and oxidative stress in the enhanced activity of ultrafines. Toxicol Appl Pharmacol. 2001;175:191-199.

26. Heslop-Harrison J, Heslop-Harrison Y. Evaluation of pollen viability by enzymatically induced fluorescence; intracellular hydrolysis of fluorescein diacetate. Stain Technol. 1970;45:115-120.

27. Pinillos V, Cuevas J. Standardization of the fluorochromatic reaction test to assess pollen viability. Biotech Histochem. 2008;83:15-21.

28. Roshchina VV, Karnaukhov VN. Changes in pollen autofluorescence induced by ozone. Biologia Plantarum. 1999;42:273-278.

29. Oberdorster E. Manufactured nanomaterials (fullerences, $\mathrm{C}_{60}$ ) induce oxidative stress in the brain of juvenile bass. Environ. Health Perspect. 2004;112:1058-1062.

30. Sayes CM, Gobin AM, Ausman KD, Mendez J, West JL, Colvin VL. Nano- $\mathrm{C}_{60}$ cytotoxicity is due to lipid peroxidation. Biomaterials. 2005;26:7587-7595
Nanotechnology, Science and Applications

\section{Publish your work in this journal}

Nanotechnology, Science and Applications is an international, peerreviewed, open access journal that focuses on the science of nanotechnology in a wide range of industrial and academic applications. It is characterized by the rapid reporting across all sectors, including engineering, optics, bio-medicine, cosmetics, textiles, resource sustainability

\section{Dovepress}

and science. Applied research into nano-materials, particles, nanostructures and fabrication, diagnostics and analytics, drug delivery and toxicology constitute the primary direction of the journal. The manuscript management system is completely online and includes a very quick and fair peer-review system, which is all easy to use. 